\title{
SUPERPOSABILITY OF STEADY AXI-SYMMETRICAL FLOWS IN A NON-NEWTONIAN FLUID
}

\author{
By (Miss) S. L. RAThNa \\ (Department of Applied Mathematics, Indian Institute of Science, Bangalore-12) \\ Received November 2, 1959 \\ (Communicated by P. L. Bhatnagar, F.N.I., F.A.sc.)
}

LET $\left(\boldsymbol{q}_{1}, p_{1}, \Omega_{1}\right)$ and $\left(\boldsymbol{q}_{2}, p_{2}, \Omega_{2}\right)$ be the two flows of an incompressible fluid of uniform density $\rho$, kinematic viscosity $\nu$ and kinematic cross-viscosity $v_{\mathfrak{c}}$, where $q, p, \Omega$ denote the velocity vector, the presssure and the potential from which the external forces are assumed to be derived. The two flows are said to be superposable, if a pressure $\left(p_{1}+p_{2}+\pi\right)$ can be found such that $\left(q_{1}+q_{2}, p_{1}+p_{2}+\pi, \Omega_{1}+\Omega_{2}\right)$ is also a solution of the Stokes-Navier equation with the necessary modification in the initial and boundary conditions.

The equations of motion for a non-Newtonian fluid are given by,

$$
\begin{aligned}
\rho\left(\frac{\partial u_{i}}{\partial t}+u_{i, j} u_{j}\right) & =t^{i}{ }_{j, j}+f^{i}, \\
u_{j, j} & =0
\end{aligned}
$$

$t_{j}{ }^{i}$ being the stress tensor given by

$$
t_{j}^{i}=-p \delta_{j}^{i}+\mathrm{F}_{1} d_{j}^{i}+\mathrm{F}_{2} d_{a}^{i} d_{j}^{a}
$$

where $f^{i}$ is the body force,

$$
d_{j}^{i}=u_{j, i}+u_{i, j}
$$

is the rate of deformation tensor, $F_{1}$ and $F_{2}$ are functions of some material constants and the second and third invariants of the rate of deformation tensor $d_{j}^{i}$.

By taking $F_{1}=\mu$ and $F_{2}=\mu_{c}$ we recognize $F_{1}$ and $F_{2}$ to be the coefficient of viscosity and coefficient of cross-viscosity.

1. For the problem on hand it is convenient to use cylindrical coordinate system $(r, \theta, Z)$ in which $z$-axis is taken to be the axis of symmetry and $r$ is the distance from the axis. The velocity components at any point 
are given by $u_{r}, u_{\theta}$ and $u_{z}$, where the velocity vector $q$ does not necessarily lie in the meridian plane, so that

$$
q=u_{r} i_{r}+u_{\theta} i_{\theta}+u_{z} i_{z}
$$

where for axial-symmetry the velocity components are functions of $r$ and $z$ only besides the time $t$.

(a) Equations of motion.-The equations of steady motion in cylindrical co-ordinates, with terms of azimuthal variation neglected, are,

$$
\begin{aligned}
& \rho\left(u_{r} \frac{\partial u_{r}}{\partial r}+u_{z} \frac{\partial u_{r}}{\partial z}-\frac{u_{\theta}^{2}}{r}\right)=\frac{\partial \mathrm{P}_{r r}}{\partial r}+\frac{\partial \mathrm{P}_{r z}}{\partial z}+\frac{\mathrm{P}_{r r}-\mathrm{P}_{\theta \theta}}{r}, \\
& \rho\left(u_{r} \frac{\partial u_{\theta}}{\partial r}+u_{z} \frac{\partial u_{\theta}}{\partial z}+\frac{u_{r} u_{\theta}}{r}\right)=\frac{\partial \mathrm{P}_{r \theta}}{\partial r}+\frac{\partial \mathrm{P}_{\theta z}}{\partial z}+\frac{2 \mathrm{P}_{r \theta}}{r}, \\
& \rho\left(u_{r} \frac{\partial u_{z}}{\partial r}+u_{z} \frac{\partial u_{z}}{\partial z}\right)=\frac{\partial \mathrm{P}_{r z}}{\partial r}+\frac{\partial \mathrm{P}_{z z}}{\partial z}+\frac{\mathrm{P}_{r z}}{r}
\end{aligned}
$$

along with the equation of continuity

$$
\frac{\partial u_{r}}{\partial r}+\frac{u_{r}}{r}+\frac{\partial u_{z}}{\partial z}=0
$$

where the stress matrix $\mathrm{P}_{i j}$ and the rate of strain matrix $e_{i j}$ in the case of non-Newtonian fluids are related as (Braun and Reiner ${ }^{1}$ )

$$
\begin{aligned}
\left(\begin{array}{c}
\mathrm{P}_{r r} \mathrm{P}_{r \theta} \mathrm{P}_{r z} \\
\mathrm{P}_{\theta r} \mathrm{P}_{\theta \theta} \mathrm{P}_{\theta z} \\
\mathrm{P}_{z r} \mathrm{P}_{z \theta} \mathrm{P}_{z z}
\end{array}\right)= & -p\left(\begin{array}{ccc}
1 & 0 & 0 \\
0 & 1 & 0 \\
0 & 0 & 1
\end{array}\right)+\mu\left(\begin{array}{c}
e_{r r} e_{r \theta} e_{r z} \\
e_{\theta r} e_{\theta \theta} e_{\theta z} \\
e_{z r} e_{z \theta} e_{z z}
\end{array}\right) \\
& +\mu_{c}\left(\begin{array}{c}
e_{r r} e_{r \theta} e_{r z} \\
e_{\theta r} e_{\theta \theta} e_{\theta z} \\
e_{z r} e_{z \theta} e_{z z}
\end{array}\right)\left(\begin{array}{c}
e_{r r} e_{r \theta} e_{r z} \\
e_{\theta r} e_{\theta \theta} e_{\theta z} \\
e_{z r} e_{z \theta} e_{z z}
\end{array}\right),
\end{aligned}
$$

where $e_{i j}=e_{j i}$

$$
\left.\begin{array}{ll}
e_{r r}=2 \frac{\partial u_{r}}{\partial r} & e_{r \theta}=\frac{\partial u_{\theta}}{\partial r}-\frac{u_{\theta}}{r} \\
e_{\theta \theta}=2 \frac{u_{r}}{r} & e_{r z}=\frac{\partial u_{r}}{\partial z}+\frac{\partial u_{z}}{\partial r} \\
e_{z z}=2 \frac{\partial u_{z}}{\partial z} & e_{\theta z}=\frac{\partial u_{\theta}}{\partial z}
\end{array}\right\}
$$

Using the relations (1.4), (1.5) and (1.6), the equations of motion (1.1), (1.2) and (1.3) for axially symmetric steady flow become, 
Superposability of Steady Axi-Symmetrical Flow's in Non-Newtonian Fluid

$$
\begin{aligned}
u_{r} \frac{\partial u_{r}}{\partial r}+ & u_{z} \frac{\partial u_{r}}{\partial z}-\frac{u_{\theta}^{2}}{r} \\
= & -\frac{1}{\rho} \frac{\partial p}{\partial r}+v\left(\nabla^{2} u_{r}-\frac{u_{r}}{r^{2}}\right)+2 v_{c}\left\{4 \frac{\partial u_{r}}{\partial r} \cdot \frac{\partial^{2} u_{r}}{\partial r^{2}}\right. \\
& +\left(\frac{\partial u_{r}}{\partial z}+\frac{\partial u_{z}}{\partial r}\right)\left(\frac{\partial^{2} u_{r}}{\partial r \partial}+\frac{\partial^{2} u_{z}}{\partial r^{2}}\right)-\frac{u_{r}}{r} \frac{\partial^{2} u_{z}}{\partial r \partial}-\frac{u_{r}}{r} \frac{\partial^{2} u_{r}}{\partial z^{2}} \\
& \left.-\frac{1}{2 r}\left(\frac{\partial u_{r}}{\partial z}\right)^{2}+\frac{1}{2 r}\left(\frac{\partial u_{z}}{\partial r}\right)^{2}+\frac{2}{r}\left(\frac{\partial u_{r}}{\partial r}\right)^{2}-\frac{2 u_{r}^{2}}{r^{3}}\right\} \\
& +v_{c}\left\{2\left(\frac{\partial u_{\theta}}{\partial r}-\frac{u_{\theta}}{r}\right) \frac{\partial}{\partial r}\left(\frac{\partial u_{\theta}}{\partial r}-\frac{u_{\theta}}{r}\right)+\frac{\partial u_{\theta}}{\partial z} \frac{\partial}{\partial z}\left(\frac{\partial u_{\theta}}{\partial r}-\frac{u_{\theta}}{r}\right)\right. \\
& \left.-\frac{1}{r}\left(\frac{\partial u_{\theta}}{\partial z}\right)^{2}+\left(\frac{\partial u_{\theta}}{\partial r}-\frac{u_{\theta}}{r}\right) \frac{\partial^{2} u_{\theta}}{\partial z^{2}}\right\}, \\
u_{r} \frac{\partial u_{\theta}}{\partial r}+ & u_{z} \frac{\partial u_{\theta}}{\partial z}+\frac{u_{r} u_{\theta}}{r} \quad(1.1 a) \\
= & -1 \frac{\partial p}{\partial \theta}+\nu\left(\nabla^{2} u_{\theta}-\frac{u_{\theta}}{r}\right)+v_{c}\left\{\frac{\partial^{2} u_{\theta}}{\partial r \partial z}\left(\frac{\partial u_{r}}{\partial z}+\frac{\partial u_{z}}{\partial r}\right)\right. \\
& +\frac{\partial u_{\theta}}{\partial z} \frac{\partial}{\partial r}\left(\frac{\partial u_{r}}{\partial z}+\frac{\partial u_{z}}{\partial r}\right)-2 \frac{\partial^{2} u_{r}}{\partial r \partial z} \frac{\partial u_{\theta}}{\partial z}+\left(\frac{\partial u_{r}}{\partial z}+\frac{\partial u_{z}}{\partial r}\right) \\
& \times \frac{\partial}{\partial z}\left(\frac{\partial u_{\theta}}{\partial r}-\frac{u_{\theta}}{r}\right)+\left(\frac{\partial u_{\theta}}{\partial r}-\frac{u_{\theta}}{r}\right) \frac{\partial}{\partial z}\left(\frac{\partial u_{r}}{\partial z}+\frac{\partial u_{z}}{\partial r}\right) \\
& -2 \frac{\partial u_{z}}{\partial z} \frac{\partial}{\partial r}\left(\frac{\partial u_{\theta}}{\partial r}-\frac{u_{\theta}}{r}\right)-2 \frac{\partial^{2} u_{\theta}}{\partial z^{2}} \frac{\partial u_{r}}{\partial r} \\
& -2 \frac{\partial^{2} u_{z}}{\partial r \partial z}\left(\frac{\partial u_{\theta}}{\partial r}-\frac{u_{\theta}}{r}\right)-\frac{4}{r} \frac{\partial u_{z}}{\partial z}\left(\frac{\partial u_{\theta}}{\partial r}-\frac{u_{\theta}}{r}\right) \\
& \left.+\frac{2}{r}\left(\frac{\partial u_{r}}{\partial z}+\frac{\partial u_{z}}{\partial r}\right) \frac{\partial u_{\theta}}{\partial z}\right\}, \\
&
\end{aligned}
$$

and

$$
\begin{aligned}
u_{r} \frac{\partial u_{z}}{\partial r}+ & u_{z} \frac{\partial u_{z}}{\partial z} \\
= & -\frac{1}{\rho} \frac{\partial p}{\partial z}+\nu \nabla^{2} u_{z}+2 v_{c}\left\{4-\frac{\partial u_{z}}{\partial z} \cdot \frac{\partial^{2} u_{z}}{\partial z^{2}}-\frac{u_{r}}{r} \frac{\partial^{2} u_{r}}{\partial r \partial z}\right. \\
& +\left(\frac{\partial u_{r}}{\partial z}+\frac{\partial u_{z}}{\partial r}\right)\left(\frac{\partial^{2} u_{r}}{\partial z^{2}}+\frac{\partial^{2} u_{z}}{\partial r \partial z}\right)-\frac{u_{r}}{r} \frac{\partial u^{2} z}{\partial r^{2}} \\
& \left.-\frac{1}{r} \frac{\partial u_{r}}{\partial r} \cdot \frac{\partial u_{r}}{\partial z}-\frac{1}{r} \frac{\partial u_{r}}{\partial r} \cdot \frac{\partial u_{z}}{\partial r}\right\}+v_{c}\left\{\frac{\partial u_{\theta}}{\partial z}\left(2 \frac{\partial^{2} u_{\theta}}{\partial z^{2}}+\frac{\partial^{2} u_{\theta}}{\partial r^{2}}\right)\right. \\
& \left.+\frac{\partial^{2} u_{\theta}}{\partial r^{2} \partial z}\left(\frac{\partial u_{\theta}}{\partial r}-\frac{u_{\theta}}{r}\right)\right\} .
\end{aligned}
$$


Expressing the velocity components in terms of the Stokes' stream function $\psi$, we have

$$
u_{r}=\frac{1}{r} \frac{\partial \psi}{\partial z}, u_{\theta}=\chi(r, z), u_{z}=-\frac{1}{r} \frac{\partial \psi}{\partial r},
$$

i.e.,

$$
q=\left(\frac{1}{r} \frac{\partial \psi}{\partial z}\right) i_{r}+x_{i_{\theta}}+\left(-\frac{1}{r} \frac{\partial \psi}{\partial r}\right) i_{z}
$$

(b) The conditions of integrability.-The conditions of integrability of the equations of motion in terms of the Stokes' stream function $\psi$ turn out to be,

$$
\begin{aligned}
& r \mathrm{~J}\left(\begin{array}{cc}
\psi & r^{-2} \mathrm{E}^{2} \psi \\
z & r
\end{array}\right)-2 \chi \frac{\partial \chi}{\partial z}
\end{aligned}
$$

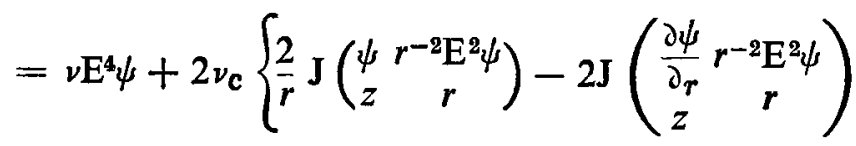

$$
\begin{aligned}
& \left.-\frac{\partial}{\partial z}\left(\frac{1}{r} \mathrm{E}^{2} \psi\right)^{2}-\frac{1}{r^{2}} \frac{\partial \psi}{\partial z} \mathrm{E}^{4} \psi+\frac{2}{r} \frac{\partial \psi}{\partial z} \frac{\partial}{\partial r}\left(\frac{\mathrm{E}^{2} \psi}{r^{2}}\right)\right\} \\
& +v_{c}\left\{r^{2 \mathrm{~J}}\left(\begin{array}{cc}
\mathrm{E}^{2} \chi & \frac{\chi}{r} \\
z & r
\end{array}\right)-2 r \mathrm{~J}\left(\begin{array}{c}
\frac{\partial \chi}{\partial r} \frac{\chi}{r} \\
z
\end{array}\right)-\frac{3 \chi}{r^{2}} \frac{\partial X}{\partial z}\right. \\
& \left.-5 \frac{\partial \chi}{\partial z} \mathrm{E}^{2 \chi}\right\}
\end{aligned}
$$

and

$$
\begin{aligned}
& \frac{1}{r^{2}} \mathrm{~J}\left(\begin{array}{cc}
\psi r & \gamma \\
z & r
\end{array}\right) \\
& =\nu\left(\nabla^{2} \chi-\frac{\chi}{r^{2}}\right)+\nu_{c}\left\{\mathrm{~J}\left(\begin{array}{cc}
\nabla^{2} \psi & \frac{\chi}{r} \\
z & r
\end{array}\right)+\frac{2}{r} \mathrm{~J}\left(\begin{array}{cc}
\frac{\partial \psi}{\partial z} & \frac{\partial \chi}{\partial z} \\
z & r
\end{array}\right)\right. \\
& \left.+\frac{2}{r} \mathrm{~J}\left(\begin{array}{c}
\frac{\partial \psi}{\partial r} \frac{\partial \chi}{\partial r} \\
z
\end{array}\right)+\frac{2}{r^{2}} \frac{\partial \psi}{\partial r} \frac{\partial^{2} \chi}{\partial r \partial z}+\frac{2}{r^{2}} \frac{\partial \psi}{\partial z} \frac{\partial^{2} \chi}{\partial z^{2}}\right\},
\end{aligned}
$$

where

$$
\mathrm{E}^{2}=\frac{\partial^{2}}{\partial r^{2}}+\frac{\partial^{2}}{\partial z^{2}}-\frac{1}{r} \frac{\partial}{\partial r}
$$


Superposability of Steady Axi-Symmetrical Flow's in Non-Newtonian Fluid

and

$$
\nabla^{2} \equiv \frac{\partial^{2}}{\partial r^{2}}+\frac{\partial^{2}}{\partial z^{2}}+\frac{1}{r} \frac{\partial}{\partial r}
$$

(c) Conditions for superposability of two motions.-Taking the two motions given by

$$
\boldsymbol{q}_{s}=\left(\frac{1}{r} \frac{\partial \psi_{s}}{\partial z}\right) i_{r}+\chi_{s} i_{\theta}+\left(-\frac{1}{r} \frac{\partial \psi_{s}}{\partial r}\right) i_{z}
$$

where $s=1,2$ corresponds to two motions. Using the conditions of integrability (1.8) and (1.9), we obtain the following conditions for superposability of two motions:

$$
\begin{aligned}
& r \mathrm{~J}\left(\begin{array}{cc}
\psi_{1} & r^{-2} \mathrm{E}^{2} \psi_{2} \\
z & r
\end{array}\right)+r \mathrm{~J}\left(\begin{array}{cc}
\psi_{2} & r^{-2} \mathrm{E}^{2} \psi_{1} \\
z & r
\end{array}\right)-2 \chi_{1} \frac{\partial \chi_{2}}{\partial z}-2 \chi_{2} \frac{\partial \chi_{1}}{\partial z} \\
& =2 \nu_{c}\left\{\frac{2}{r} \mathrm{~J}\left(\begin{array}{cc}
\psi_{1} r^{-2} \mathrm{E}^{2} \psi_{2} \\
z & r
\end{array}\right)+\frac{2}{r} \mathrm{~J}\left(\begin{array}{cc}
\psi_{2} r^{-2} \mathrm{E}^{2} \psi_{1} \\
z & r
\end{array}\right)\right. \\
& -2 \mathrm{~J}\left(\begin{array}{cc}
\frac{\partial \psi_{1}}{\partial r} & r^{-2} \mathrm{E}^{2} \psi_{2} \\
z & r
\end{array}\right)-2 \mathrm{~J}\left(\begin{array}{cc}
\frac{\partial \psi_{2}}{\partial r} & r^{-2} \mathrm{E}^{2} \psi_{1} \\
z & r
\end{array}\right) \\
& +\frac{2}{r} \frac{\partial \psi_{1}}{\partial z} \frac{\partial}{\partial r}\left(\frac{E^{2} \psi_{2}}{r^{2}}\right)+\frac{2}{r} \frac{\partial \psi_{2}}{\partial z} \frac{\partial}{\partial r}\left(\frac{E^{2} \psi_{1}}{r^{2}}\right)-\frac{1}{r^{2}} \frac{\partial \psi_{1}}{\partial z} \mathrm{E}^{4} \psi_{2} \\
& \left.-\frac{1}{r^{2}} \frac{\partial \psi_{2}}{r z} \mathrm{E}^{4} \psi_{1}\right\}+v_{\mathrm{c}}\left\{r^{2} \mathrm{~J}\left(\begin{array}{cc}
\mathrm{E}^{2} \chi_{1} & \frac{\chi_{2}}{r} \\
z & r
\end{array}\right)+r^{2 \mathrm{~J}}\left(\begin{array}{cc}
\mathrm{E}^{2} \chi_{2} & \frac{\chi_{1}}{r} \\
z & r
\end{array}\right)\right. \\
& -2 r \mathrm{~J}\left(\begin{array}{cc}
\frac{\partial \chi_{1}}{\partial r} & \chi_{2} \\
z & r
\end{array}\right)-2 r \mathrm{~J}\left(\begin{array}{cc}
\frac{\partial \chi_{2}}{\partial r} & \frac{\chi_{1}}{r} \\
z & r
\end{array}\right)-\frac{3 \chi_{1}}{r^{2}} \frac{\partial \chi_{2}}{\partial z} \\
& \left.-3 \frac{\chi_{2}}{r^{2}} \frac{\partial \chi_{1}}{\partial z}-5 \frac{\partial \chi_{1}}{\partial z} \mathrm{E}^{2} \chi_{2}-5 \frac{\partial \chi_{2}}{\partial z} \mathrm{E}^{2} \chi_{1}\right\}
\end{aligned}
$$

and

$$
\begin{aligned}
& \mathrm{J}\left(\begin{array}{cc}
\psi_{1} & r \chi_{2} \\
z & r
\end{array}\right)+\mathrm{J}\left(\begin{array}{cc}
\psi_{2} & r \chi_{1} \\
z & r
\end{array}\right) \\
& \quad=\frac{\nu_{c}}{r}\left\{\mathrm{~J}\left(\begin{array}{cc}
\nabla^{2} \psi_{1} & \frac{\chi_{2}}{r} \\
z & r
\end{array}\right)+\mathrm{J}\left(\begin{array}{cc}
\nabla^{2} \psi_{2} & \frac{\chi_{1}}{r} \\
z & r
\end{array}\right)+2 \mathrm{~J}\left(\begin{array}{cc}
\frac{\partial \psi_{1}}{\partial z} & \frac{\partial \chi_{2}}{\partial z} \\
z & r
\end{array}\right)\right.
\end{aligned}
$$




$$
\begin{aligned}
& +2 \mathrm{~J}\left(\begin{array}{cc}
\frac{\partial \psi_{2}}{\partial z} & \frac{\partial X_{1}}{\partial z} \\
z & r
\end{array}\right)+2 \mathrm{~J}\left(\begin{array}{cc}
\frac{\partial \psi_{1}}{\partial r} & \frac{\partial X_{2}}{\partial r} \\
z & r
\end{array}\right)+2 \mathrm{~J}\left(\begin{array}{cc}
\frac{\partial \psi_{2}}{\partial r} & \frac{\partial \chi_{1}}{\partial r} \\
z & r
\end{array}\right) \\
& \left.+\frac{2}{r} \frac{\partial \psi_{1}}{\partial r} \frac{\partial^{2} \chi_{2}}{\partial r \partial z}+\frac{2}{r} \frac{\partial \psi_{2}}{\partial r} \frac{\partial^{2} \chi_{1}}{\partial r \partial z}+\frac{2}{r} \frac{\partial \psi_{1}}{\partial z} \frac{\partial^{2} \chi_{2}}{\partial z^{2}}+\frac{2}{r} \frac{\partial \psi_{2}}{\partial z} \frac{\partial^{2} \chi_{1}}{\partial z^{2}}\right\} .
\end{aligned}
$$

We can easily show that the conditions (1.10) and (1.11) are both necessary and sufficient for the two axially symmetric flows of a non-Newtonian fluid corresponding to the two stream functions $\psi_{1}$ and $\psi_{2}$ to be superposable.

\section{CONDITIONS FOR SELF-SUPERPOSABILITY OF A FLOW:}

Using the conditions (1.10) and (1.11), it follows that the flow with stream function $\psi$ is self-superposable if and only if,

$$
\begin{aligned}
& r \mathrm{~J}\left(\begin{array}{cc}
\psi r^{-2} \mathrm{E}^{2} \psi \\
z & r
\end{array}\right)-2 \chi \frac{\partial \chi}{\partial z}
\end{aligned}
$$

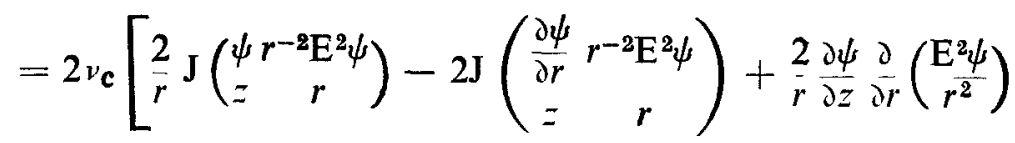

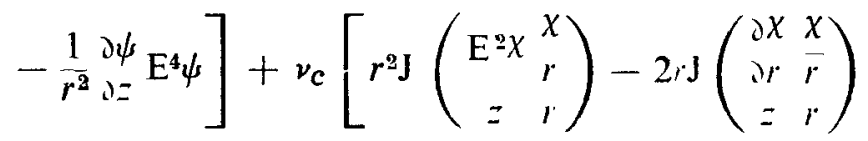

$$
\begin{aligned}
& \left.-\frac{3 x}{r^{2}} \frac{\partial X}{\partial z}-5 \frac{\partial X}{\partial z} \mathrm{E}^{2} x\right]
\end{aligned}
$$

and

$$
\begin{aligned}
\mathrm{J}\left(\begin{array}{cc}
\psi r & r \\
z & r
\end{array}\right)=\frac{\nu_{\mathrm{c}}}{r} & {\left[\mathrm{~J}\left(\begin{array}{cc}
\nabla^{2} \psi & \frac{\chi}{r} \\
z & r
\end{array}\right)+2 \mathrm{~J}\left(\begin{array}{cc}
\frac{\partial \psi}{\partial z} & \frac{\partial \chi}{\partial z} \\
z & r
\end{array}\right)+2 \mathrm{~J}\left(\begin{array}{cc}
\frac{\partial \psi}{\partial r} & \frac{\partial \chi}{\partial r} \\
z & r
\end{array}\right)\right.} \\
& \left.+\frac{2}{r} \frac{\partial \psi}{\partial r} \frac{\partial^{2} \chi}{\partial r \partial z}+\frac{2}{r} \frac{\partial \psi}{\partial z} \frac{\partial^{2} \chi}{\partial z^{2}}\right] .
\end{aligned}
$$

\section{Particular Cases}

(a) Newtonian fluids.-If in the conditions of superposability and selfsuperposability by putting $\nu_{c}=0$, we obtain the conditions of superposability 
uperposability of Steady Axi-Symmetrical Flows in Non-Newtonian Fluid 161 and self-superposability for a Newtonian fluid as obtained by Lakshmana Rao. $^{2}$

(b) Velocity vector lies in the meridian plane.-We can find the conditions of superposability and self-superposability in steady axi-symmetrical flows when the velocity vector $q$ lies in the meridian plane, i.e.,

$$
q=u_{r} i_{r}+u_{z} i_{z}
$$

or in terms of the Stokes' stream function

$$
q=\left(\frac{1}{r} \frac{\partial \psi}{\partial z}\right) i_{r}+\left(-\frac{1}{r} \frac{\partial \psi}{\partial r}\right) i_{z}
$$

and the vorticity is given by

$$
\omega=\frac{1}{r} \mathrm{E}^{2} \psi \text {. }
$$

In this case the equations of motion are given by $(1.1 a),(1.2 a)$ and (1.3a) with $\chi=0$.

The condition of integrability (1.8) becomes

$$
\begin{aligned}
& r J\left(\begin{array}{cc}
\psi & r^{-2} E^{2} \psi \\
z & r
\end{array}\right)
\end{aligned}
$$

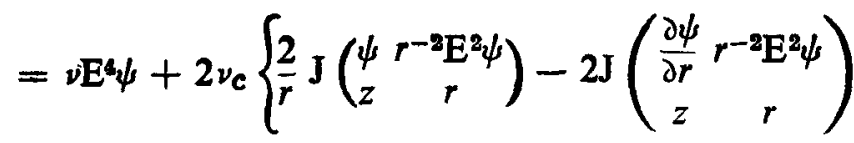

$$
\begin{aligned}
& \left.-\frac{1}{r^{2}} \frac{\partial \psi}{\partial z} \mathrm{E}^{4} \psi-\frac{\partial}{\partial z}\left(\frac{1}{r} \mathrm{E}^{2} \psi\right)^{2}+\frac{2}{r} \frac{\partial \psi}{\partial z} \frac{\partial}{\partial r}\left(\frac{\mathrm{E}^{2} \psi}{r^{2}}\right)\right\}
\end{aligned}
$$

while the condition (1.9) is automatically satisfied.

The condition of superposability (1.10) for two axi-symmetrical flows with stream functions $\psi_{1}$ and $\psi_{2}$ is given by,

$$
\begin{aligned}
& \mathrm{J}\left(\begin{array}{cc}
\psi_{1} & r^{-2} \mathrm{E}^{2} \psi_{2} \\
z & r
\end{array}\right)+\mathrm{J}\left(\begin{array}{cc}
\psi_{2} & r^{-2} \mathrm{E}^{2} \psi_{1} \\
z & r
\end{array}\right) \\
& =\frac{2 \nu_{c}}{r}\left\{\frac{2}{r} \mathrm{~J}\left(\begin{array}{cc}
\psi_{1} & r^{-2} \mathrm{E}^{2} \psi_{2} \\
z & r
\end{array}\right)+\frac{2}{r} \mathrm{~J}\left(\frac{\psi_{2}}{z} \underset{r}{r-2} \mathrm{E}^{2} \psi_{1}\right)\right. \\
& -2 \mathrm{~J}\left(\begin{array}{cc}
\frac{\partial \psi_{1}}{\partial r} & r^{-2} \mathrm{E}^{2} \psi_{2} \\
z & r
\end{array}\right)-2 \mathrm{~J}\left(\begin{array}{cc}
\frac{\partial \psi_{2}}{\partial r} & r^{-2} \mathrm{E}^{2} \psi_{1} \\
z & r
\end{array}\right)
\end{aligned}
$$




$$
\begin{aligned}
& -\frac{1}{r^{2}} \frac{\partial \psi_{1}}{\partial z} \mathrm{E}^{4} \psi_{2}-\frac{1}{r^{2}} \frac{\partial \psi_{2}}{\partial z} \mathrm{E}^{4} \psi_{1}+\frac{2}{r} \frac{\partial \psi_{1}}{\partial z} \frac{\partial}{\partial r}\left(\frac{\mathrm{E}^{4} \psi_{2}}{r^{2}}\right) \\
& \left.+\frac{2}{r} \frac{\partial \psi_{2}}{\partial z} \frac{\partial}{\partial r}\left(\frac{\mathrm{E}^{4} \psi_{1}}{r^{2}}\right)\right\} .
\end{aligned}
$$

The condition (1.11) is automatically satisfied. The condition for self-superposability of this flow is given by:

$$
\begin{gathered}
\mathrm{J}\left(\begin{array}{cc}
\psi r^{-2} \mathrm{E}^{2} \psi \\
z & r
\end{array}\right) \\
=\frac{2{ }_{c}}{r}\left\{\frac{2}{r} \mathrm{~J}\left(\begin{array}{cc}
\psi r^{-2} \mathrm{E}^{2} \psi \\
z & r
\end{array}\right)-2 \mathrm{~J}\left(\begin{array}{cc}
\frac{\partial \psi}{\partial r} & r^{-2} \mathrm{E}^{2} \psi \\
z & r
\end{array}\right)\right. \\
-\frac{1}{r^{2}}\left(\frac{\partial \psi}{\partial z} \mathrm{E}^{4} \psi+\frac{2}{r} \frac{\partial \psi}{\partial z} \frac{\partial}{\partial r}\left(\frac{\mathrm{E}^{2} \psi}{r^{2}}\right)\right\} .
\end{gathered}
$$

From the condition (3.5), we see that a rotational flow with suffix 2, is superposable on an irrotational flow with suffix 1 , if

$$
\begin{gathered}
\mathrm{J}\left(\begin{array}{cc}
\psi_{1} & r^{-2} \mathrm{E}^{2} \psi_{2} \\
z & r
\end{array}\right) \\
=\frac{2 v_{\mathrm{c}}}{r}\left\{\frac{2}{r} \mathrm{~J}\left(\begin{array}{cc}
\psi_{1} & r^{-2} \mathrm{E}^{2} \psi_{2} \\
z & r
\end{array}\right)-2 \mathrm{~J}\left(\begin{array}{cc}
\frac{\partial \psi_{1}}{\partial r} r^{-2} \mathrm{E}^{2} \psi_{2} \\
z & r
\end{array}\right)\right. \\
\left.-\frac{1}{r^{2}} \frac{\partial \psi_{1}}{\partial z} \mathrm{E}^{4} \psi_{2}+\frac{2}{r} \frac{\partial \psi_{2}}{\partial z} \frac{\partial}{\partial r}\left(\frac{\mathrm{E}^{2} \psi_{2}}{r^{2}}\right)\right\} .
\end{gathered}
$$

Using the conditions (3.5) and (3.6), we can prove the following theorems.

THEOREM 1.-All irrotational motions are self-superposable and any two irrotational motions are superposable on each other.

THEOREM 2.-The two flows will be superposable if the vorticity of each flow is proportional to r. Also an axi-symmetrical flow will be self-superposable if its vorticity is proportional to $\mathrm{r}$.

THSOREM 3.-If $\omega_{1} / \mathrm{r}$ is constant along the stream lines of flow 2, while $\omega_{2} / \mathrm{r}$ is constant along the stream lines of flow 1 , then the two flows are superposable if in addition the velocity components and vorticities satisfy the condition

$$
2\left(u_{1} v_{1}+u_{2} v_{2}\right)+r\left(v_{1} \omega_{1}+v_{2} \omega_{2}\right)=0 .
$$

(where $\omega_{1}$ and $\omega_{2}$ are the vorticities of the two flows). 
Superposability of Steady Axi-Symmetrical Flows in Non-Newtonian Fluid 163

Theorem 4.-An axi-symmetrical flow is self-superposable, if the vorticity $\omega$ is of the form $\omega=\mathrm{f}(\psi) \mathrm{r}$ and if the stream function $\psi$ satisfies the condition

$$
E^{4} \psi=2 \mathrm{r} \frac{\partial}{\partial \mathrm{r}}\left(\frac{1}{\mathrm{r}^{2}} E^{2} \psi\right)
$$

While the condition of integrability reduces to

$$
\nu \mathrm{E}^{4} \psi=\frac{2 v_{\mathrm{c}}}{r^{2}} \frac{\partial}{\partial z}\left(\mathrm{E}^{4} \psi\right)
$$

Also a rotational flow with stream function $\psi_{2}$ is superposable on an irrotational flow with stream function $\psi_{1}$, if in general

$$
\omega_{2}=f\left(\psi_{1}\right) r
$$

and

$$
\mathrm{E}^{4} \psi_{2}=2 r \frac{\partial}{\partial r}\left(\frac{1}{r^{2}} \mathrm{E}^{2} \psi_{2}\right)
$$

The corresponding theorems for a Newtonian fluid have been given by Bhatnagar and Verma. ${ }^{3}$

I am deeply grateful to Prof. P. L. Bhatnagar for suggesting the problem and for his kind guidance throughout this work.

\section{REFERENCES}

1. Braun and Reiner, M. .. Quar. Jour. Mech. and App. Math., 1952, 5, 42-53.

2. Lakshmana Rao, S. K. .. Proc. Ind. Acad. Sci., 1957, 45 A, 418-23.

3. Bhatnagar, P. L. and Verma, Ibid., 1957, 45 A, 281-92.

P. D. 